\title{
Efecto de un programa de Mindfulness sobre variables motivacionales y psicológicas en educación Primario
}

Gonzálo Almansa, $M^{a}$ Araceli Budía, José Luis López, $M^{a}$ José Márquez, Ana Isabel Martínez, Benjamín Palacios, $M^{a}$ del Mar Peña, Pilar Santafé, Jesús Zafra, Eduardo Fernández-Ozcorta, Pedro Sáenz-López

\section{Email: psaenz@uhu.es}

\section{RESUMEN:}

El mindfulness es una técnica de meditación que consiste en tener atención plena hacia el momento presente. Hay cada vez más evidencias científicas de sus beneficios educativos, por lo que se planteó diseñar un programa a través de audios de alrededor de 10 minutos de duración para aplicarlos en el tercer ciclo de primaria de dos colegios públicos de Huelva durante 7 semanas. La muestra fue de 157 alumnos divididos en dos grupos control y dos grupos experimentales. Se aplicaron unos cuestionarios sobre variables motivaciones (motivación, orientaciones metas, necesidades psicológicas básicas) y emocionales (bienestar psicológico y atención). Los resultados mostraron una correlación positiva de la orientación a la tarea y el bienestar psicológico con el resto de variables. Tras la intervención, la atención mejoró en los grupos experimentales.

PALABRAS CLAVE: mindfulness, intervención, educación primaria. 


\section{INTRODUCCIÓN}

Los niños de hoy en día son nerviosos, se distraen con facilidad y pierden la atención de forma constante. Todo ello se debe a la gran variedad de estímulos que les rodean, tanto en el contexto escolar como en su día a día y esto hace que pierdan la atención y la concentración fácilmente. Padres y docentes se quejan de este problema que en multitud de ocasiones se refleja con estas frases: "Se distrae mucho en clase", "no atiende cuando se le habla", "es muy despistado". Todo esto se resumen en un único concepto: falta de atención.

Con lo que respecta al ámbito escolar, la práctica del mindfulness (atención plena) se perfila como una poderosa herramienta educativa para mejorar este déficit. Por tanto, se considera necesario realizar investigaciones en la escuela que demuestren los posibles beneficios. Hay evidencias científicas sobre los beneficios que el mindfulness tiene sobre algunos de estos conceptos. Investigaciones anteriores, afirman que la actividad mental propia de la práctica de mindfulness es capaz de cambiar significativamente el cerebro.

Según Simón (2007) "la concentración de la atención momento a momento, que al principio exige un esfuerzo considerable, (persistir, por ejemplo, en la atención a la respiración, evitando constantemente la intrusión de pensamientos o emociones), con el tiempo se va convirtiendo en un hábito automático, que no requiere apenas esfuerzo. Esta transición desde la atención al presente con esfuerzo, a la atención al presente sin esfuerzo es lo que separa al principiante del meditador experimentado".

Por ello, los objetivos planteados para esta investigación podemos resumirlos en:

- Diseñar un programa de intervención de mindfulness para tercer ciclo de primaria.

- Conocer la relación que existe entre las orientaciones motivacionales, el bienestar psicológico, las necesidades psicológicas básicas, el disfrute y la atención.

- Analizar la influencia del programa en las variables estudiadas.

En cuanto a los beneficios del mindfulness, Kabat-Zinn (1994) afirma que el mindfulness es "prestar atención de una manera especial: intencionadamente, en el momento presente y sin juzgar". Según Vallejo (2006), "mindfulness es un término que no tiene una palabra correspondiente en castellano. Puede entenderse como atención y conciencia plena, presencia atenta y reflexiva. Simón (2007) indica que es algo muy simple y familiar, algo que todos nosotros hemos experimentado en ocasiones de nuestra vida cotidiana. Cuando somos conscientes de lo que estamos haciendo, pensando o sintiendo, estamos practicando mindfulness. Lo que sucede es que habitualmente nuestra mente se encuentra vagando sin orientación alguna, saltando de unas imágenes a otras, de unos a otros pensamientos. Mindfulness es una capacidad humana universal y básica, que consiste en la posibilidad de ser conscientes de los contenidos de la mente momento a momento. Es la práctica de la autoconciencia. El mindfulness no es un descubrimiento moderno, aunque vivamos ahora su redescubrimiento ( $\mathrm{y}$ un cierto reencuentro) en el marco de la cultura 
occidental. Esta técnica representa el corazón de las enseñanzas originales de Siddhartha Gautama Sakyamuni, popularmente más conocido como Buda (563 a. C.).

El origen del mindfulness es el Budismo, concretamente el Budismo Theravada, por lo que mindfulness tiene más de 25 siglos de antigüedad. El principal objetivo de las enseñanzas de Buda fueron, y continúan siendo, la erradicación o cese del sufrimiento humano (e.g., Fulton y Sieguel, 2005; Sogyal, 1994). La meditación o mindfulness, ideada y transmitida por Buda, constituye el método o la vía para la consecución de dicho objetivo; mindfulness es considerado como "el corazón" de la meditación budista (e.g., Nianaponika, 1962; Silananda, 1990). La antiquísima meditación vipassana (meditación originaria del Budismo y más conservadora), está siendo utilizada por la Psicología, y otras ciencias de la salud, bajo la rúbrica de meditación mindfulness. Se presenta a la psicología, y muy especialmente a la psicología Clínica, como una alternativa terapéutica, como una nueva técnica de intervención o método clínico o como una filosofía de intervención, e incluso de vida. Por lo consiguiente decimos que la meditación o mindfulness genera autoconocimiento, es decir, facilita la discriminación de nuestro propio comportamiento (Mañas, 2009; Kaiser, 2013).

Mindfulness significa prestar atención de manera consciente a la experiencia del momento con interés, curiosidad y aceptación. Este tipo de 'atención o conciencia' nos permite aprender de forma directa con aquello que está ocurriendo en el aquí y ahora, es decir, pretende entrenar a los sujetos a fin de que puedan identificar en cada instante sus propias sensaciones, emociones y pensamientos. Su práctica es, en cierto sentido, similar a la meditación. Mindfulness es un método para sosegar la mente, siendo su objeto de estudio las sensaciones, emociones, ideas... que nos ocurran en el presente. Calmar la mente para ver con claridad, sacándola de su piloto automático (Moñiva, Garcia-Diex, García-de Silva 2012).

Desde un punto de vista científico, podemos definir mindfulness como un estado en el que el practicante es capaz de mantener la atención centrada en un objeto por un periodo de tiempo teóricamente ilimitado. (Lutz, Rawlings y Davidson, 2005). O, siguiendo a Thich Naht Hanh, mindfulness es mantener viva la conciencia en la realidad del presente. La palabra deriva de un vocablo inglés que significa pali "sati" que denota conciencia, atención, recuerdo (Siegel, 2010). La atención y aceptación de la respiración, sentidos, sentimientos y del cuerpo además de liberarnos de los pensamientos viviendo el ahora son los pilares del mindfullness. (Snel, 2013).

Las investigaciones básicas del mindfulness, plantea cuestiones sobre la naturaleza de la mente y de cómo esta se relaciona con nuestros procesos cognitivos, el cerebro, el cuerpo como un todo, denominado "el yo". La investigación y aplicación práctica de mindfulness tiene lugar en materias como las neurociencias, la medicina, la psicología, el trabajo social y la psicoterapia.

Como constructo, da lugar a una teoría, que subyace una epistemología, un método y objeto de estudio y un amplio campo de investigaciones. La aplicación de la técnica del mindfulness nos permite "estar" en el momento presente, prestando 
atención al cuerpo y superando la disociación mente-cuerpo (Sánchez Gutiérrez, 2011).

Mindfulness puede estar relacionado con otros constructos como la metacognición, conciencia reflexiva y aceptación, habiendo cuestionarios para ser medido, aunque la mayor fiabilidad la aportan, como apuntan Siegel (2010) y Davidson (2012), mediante la práctica. Consiguiendo así desarrollar capacidades que se integran como nuevo recurso interno, que nos permite ser conscientes de los patrones reactivos automáticos y evolucionar hacia una relación con la experiencia menos cotidiana y más genuina, aportando al sujeto la capacidad de elaborar respuestas de calidad ante los estímulos. Esto nos aporta repercusiones directas en sus niveles de salud y en sentimiento de bienestar y felicidad.

Si vemos el mindfulness como un proceso psicológico, supone focalizar nuestra atención en lo que estamos haciendo o sintiendo, para ser conscientes del devenir de nuestro organismo y conductas. Considerando la mente como un sexto sentido.

La práctica nos aporta un cambio respecto a nosotros mismos y a nuestro contexto interno, las habilidades que se desarrollan son la empatía, compasión, simpatía, paciencia, aceptación, evitar juzgar, etc. Los beneficios para cualquier persona pueden ser: reducir las distracciones o aumentar la concentración, minimizar los efectos negativos de la ansiedad, aceptar la realidad tal y como es, disfrutar del momento presente, potenciar nuestra autoconciencia, reducir el sufrimiento y evitar o reducir la impulsividad. Por lo tanto, esta Inteligencia Emocional Plena puede ser también una herramienta eficaz para la prevención y resolución de conflictos en equipos docentes y alumnado, ya que con la práctica de mindfulness desarrollamos la capacidad de concentración de nuestra mente, lo que nos ayuda a serenarnos y a percibir, comprender y gestionar mucho mejor los desafíos que nos plantea constantemente la convivencia escolar.

Los estudios que se han ido presentando en los últimos años, tanto dentro como fuera del contexto escolar, señalan que a medida que los niños y jóvenes van adquiriendo competencias emocionales, se observa en su comportamiento las consecuencias positivas que esto pueda tener y además, evidencian que las competencias emocionales pueden contribuir de forma positiva al bienestar personal y social del alumnado (Bisquerra y Pérez, 2007)

También existen evidencias empíricas para afirmar que los alumnos emocionalmente inteligentes, como norma general, poseen mejores niveles de ajuste psicológico y bienestar emocional; presentan una mayor calidad y cantidad de redes interpersonales y de apoyo social; son menos propensos a realizar comportamientos disruptivos, agresivos o violentos; pueden llegar a obtener un mayor rendimiento escolar al enfrentarse a las situaciones de estrés con mayor facilidad y consumen menor cantidad de sustancias adictivas (e.g. tabaco, alcohol, etc.) (Extremera y Fernández-Berrocal, 2003). 
En el curso 2008-2009, el Equipo de formación e investigación INEP realizó un primer estudio sobre los efectos de un programa de entrenamiento de la Inteligencia Emocional (IE) a través de la Atención Plena con alumnos de Primaria (de 9 a 12 años) de cinco CEIPs de Málaga capital. Entre sus conclusiones, se evidenció como las competencias emocionales son predictores, no sólo del nivel de IE del alumno, sino de su bienestar psicológico; lo que determina la importancia del aprendizaje de competencias y habilidades emocionales como prioridad básica en la formación y educación de cualquier persona (familia, centros educativos, universidad, recursos ciudadanos, etc.). Los resultados obtenidos demostraron la valía de Mindfulness como instrumento para mejorar la satisfacción vital y la realidad emocional en niños preadolescentes. A su vez, han señalado al programa como una herramienta útil en el refuerzo, guía y desarrollo de una empatía sana en niños, especialmente en su dimensión emocional, así como para la mejora de la atención con la disminución de pensamientos intrusivos y recurrentes.

\section{METODOLOGÍA}

Se aplicó un programa de intervención de mindfulness en tercer ciclo de primaria. Se seleccionaron dos grupos experimentales y dos grupos de control en dos colegios de la provincia de Huelva. A todo el alumnado se aplicó el cuestionario antes y después de la intervención.

\section{Participantes}

La muestra del estudio estuvo compuesta por un total de 157 alumnos, de los cuales $46,7 \%$ eran chicas y el $53,3 \%$ chicos, de edades comprendidas entre los 10 y los 12 años $(10,61 \pm, 645)$. La muestra fue recogida en dos centros escolares de diferentes localidades Onubenses. CEIP "La Noria" de Lepe y CEIP "EI Puntal" de Aljaraque, correspondiente al tercer ciclo de primaria (quinto y sexto de primaria). Cabe destacar que los dos centros escolares participantes colaboraron voluntariamente en el desarrollo de la investigación.

\section{Instrumentos}

Se emplearon diversas escalas de tipo Likert que oscila de 1 (totalmente en desacuerdo) a 5 (totalmente de acuerdo).

Intrinsic Motivation Inventory (IMI). Se empleó la versión testada en el contexto español traducida por García Calvo (2004) del cuestionario de Intrinsic Motivation Inventory (McAuley, Duncan, y Tammen, 1989). Esta subescala corresponde con tres ítems del factor del componente Diversión del cuestionario original. Presenta un valor alpha de Cronbach de .80 y está compuesto por cinco ítems.

Escala de Satisfacción con la Vida (SLWLS). Se empleó la versión testada en el contexto español traducida por Atienza, Pons, Balanguer, y García-Merita (2000) del cuestionario Escala de Satisfacción con la Vida (Diener et al, 1985), que mide la satisfacción con la vida como un proceso de juicio cognitivo, a través de 5 ítems. En este caso, se excluye el último ítem. Este factor está encabezado por el enunciado «En relación con tu vida en general...». 
Subescala de Autovalía del Cuestionario de Autodescripción (SDQ-III). Para medir la autoestima Balaguer et al. (2008) de la Subescala de Autovalía del Cuestionario de Autodescripción (SDQ-III; Marsh, Richards, Johnson, Roche y Tremayne, 1994). Esta subescala está formada por doce ítems, de los cuales hemos escogido los ítems 1, 6, 8 y 12. Este factor está encabezado por el enunciado «En relación contigo mismo...»

Escala de las Necesidades Psicológicas Básicas en el Ejercicio (BPNES). Se utilizó la adaptación al español a la educación física de la escala de las necesidades psicológicas básicas en el ejercicio traducido por Moreno, González-Cutre, Chillón, y Parra (2008) de la Escala de las Necesidades Psicológicas Básicas en el Ejercicio. El inventario consta de doce ítems agrupados en tres dimensiones (cuatro ítems por dimensión): autonomía (1, 4, 7 y 10), competencia (2, 5, 8 y 11) y relación con los demás (3, 6, 9 y 12). La consistencia interna puede ser marginalmente aceptada (Hair et al., 1998) dado el reducido número de ítems que componen los factores.

Escala de medida de las orientaciones de metas en el ejercicio (GOES). Se empleó la versión testada en el contexto español traducida por Moreno, López, Martínez Galindo, Alonso, y González- Cutre (2007). Validación preliminar de la escala de percepción del clima motivacional de los iguales (CMI) y la escala de las orientaciones de meta en el ejercicio (GOES). Es esta escala se miden dos factores: ítems relacionados con la orientación tarea $(1,4,5,7$, y 9$)$ y ítems relacionados con la orientación al ego (2, 3, 6, 8 y 10).

Escala de Atención del perfil emocional de tu cerebro. Se empleó la escala del perfil emocional de tu cerebro creada por Davidson (2012). Es una escala de verdadero o falso. La puntuación en esta escala se determina de la siguiente manera: Un punto si responde $V$ en los ítems 1, 2, 3, 6, 7 y 10 y un punto si responde $F$ en los ítems 4, 5, 8 y 9. Una vez recogidos los puntos la evaluación de esta escala está fijada en: De 0 a 3 un perfil atencional disperso; de 4 a 7 un perfil atencional intermedio y de 8 a 10 un perfil atencional centrado.

\section{Procedimiento}

Para poder realizar la recogida de datos se contactó con ambos colegios. Tras una previa reunión informativa y explicativa con el equipo directivo de los colegios implicados, se realizó una reunión a nivel de ciclo (tercer) dónde se informa sobre el concepto de mindfulness y como trabajarlo a nivel de aula.

Debido a la minoría de edad de los participantes, antes de iniciar con la intervención se les solicitó una autorización por escrito firmada por sus padres para participar en el estudio.

El programa de intervención consistió en realizar mindfulness a diario a través de audios guiados durante 7 semanas en los grupos experimentales (un grupo de 5o y otro de $6^{\circ}$ en cada colegio). Los audios fueron aportados por el equipo investigador que el profesorado puso cada día, fueron dados con anticipación y agrupados de manera semanal, analizados por dicho equipo, siendo un total de siete semanas. Hubo 
un grupo de control y otro experimental correspondiendo a las diferentes líneas de cada nivel, para proporcionar la comparación de datos.

\section{Análisis de datos}

El análisis de los resultados del estudio tuvo dos fases claramente diferencias. En una primera fase analizaron los datos surgidos de la primera toma de datos de ambos grupos. En ellos se realizó un análisis descriptivo de los grupos y comprobar que no existían diferencias estadísticamente significativas entre los grupos previo a la intervención. A su vez, se analizaron los grupos de forma global, sin diferenciar grupos, y obtener las correlaciones entre las variables estudiadas. El segundo paso, se centró en analizar los resultados de ambos grupos tras la intervención para ello se analizaron las diferencias inter e intragrupal. Se empleó un análisis T para muestra dependiente para conocer las diferencias intragrupal. Mientras que para conocer las diferencias entre grupos se recurrió a la prueba $T$ para muestras independientes. Los análisis se llevaron a cabo con el paquete estadístico SPSS 20.0. El nivel de significación estadístico empleado fue de 0.05 .

\section{RESULTADOS}

\section{Análisis descriptivos y correlaciones bivariadas}

En la Tabla 1 se presentan los estadísticos descriptivos (medias, desviaciones típicas y correlaciones) de las variables sometidas a estudio. Como se muestra en la tabla, los datos obtenidos en el pretest de toda la muestra indican que la orientación a la tarea tiene una media muy superior al ego. La diversión tiene una media muy alta, similar al clima tarea. Con relación a las necesidades psicológicas básicas, el factor "Relaciones" es el que alcanza más media y muy cerca "percepción de competencia".

Tabla. 1. Estadísticos descriptivos y correlaciones bivariadas de las variables de estudio

\begin{tabular}{|c|c|c|c|c|c|c|c|c|c|c|c|}
\hline & $\mathrm{M}$ & DT & 1 & 2 & 3 & 4 & 5 & 6 & 7 & 8 & 9 \\
\hline 1. Diversión & 4,66 & 49 & - & ,283** &, $430^{* *}$ &, $541^{* *}$ &, $434^{* *}$ &, $394^{* *}$ &, $478^{* *}$ & & \\
\hline 2. Satisfacción & 4,19 & ,73 & & &, $596^{* *}$ &, $428^{* *}$ &, $598^{* *}$ &, $458^{* *}$ &, $431^{* *}$ & & \\
\hline 3. Autovalía & 4,38 &, 58 & & & - &, $523^{* *}$ &, $662^{* *}$ &, $409^{* *}$ &, $383^{* *}$ &, $314^{* *}$ &, $177^{*}$ \\
\hline 4. Autonomía & 3,95 & ,70 & & & & - &, $535^{* *}$ &, $353^{* *}$ &, $402^{* *}$ & $273^{* *}$ & ,075 \\
\hline 5. Competencia & 4,42 & 65 & & & & & - &, $422^{* *}$ &, $522^{* *}$ &, $246^{* *}$ &, $195^{*}$ \\
\hline 6. Relaciones & 4,50 & ,60 & & & & & & - &, $421^{* *}$ &, $159^{*}$ & \\
\hline 7. Tarea & 4,67 &, 48 & & & & & & & - &, $175^{*}$ &, $183^{*}$ \\
\hline 8. Ego & 2,94 & 1,16 & & & & & & & & - & \\
\hline 9. Atención & 4,68 & 1,80 & & & & & & & & & \\
\hline
\end{tabular}

Notas. M: media; DT: desviación típica; $* p<.01 ; * p<.05$

En cuanto a las correlaciones entre variables, observamos en la tabla 1 una fuerte relación entre la mayoría. La variable de orientación a la tarea está relacionada significativamente con el resto de variables. El bienestar psicológico (Satisfacción y autovalía) correlaciona significativamente con las tres necesidades psicológicas 
básicas, orientación a la tarea y con la diversión. La variable de diversión correlaciona con las tres necesidades básicas, bienestar psicológico y la orientación a la tarea. El perfil atencional muestra una relación significativa con las variables autovalía, competencia y orientación a la tarea.

\section{Análisis de las diferencias entre pre-test y post-test}

En la tabla 2, se presentan las medias de los dos grupos previa a la intervención y tras la misma. Podemos observar que ambos grupos presentan medias más altas tras el periodo de intervención.

Tras la intervención, en grupo control existe un cambio sobre la variable Atención ya que es la que presenta mayor media, por delante de Diversión y Tarea. Este mismo acontecimiento se produce en el grupo experimental. La atención se muestra como la variable más fuerte seguida por Tarea y Competencia.

Tabla 2.- Estadísticos descriptivos pre-test y post-test

\begin{tabular}{lllll} 
& \multicolumn{2}{c}{ Control } & \multicolumn{2}{c}{ Experimental } \\
& \multicolumn{1}{c}{ Pre } & \multicolumn{1}{c}{ Post } & \multicolumn{1}{c}{ Pre } & Post \\
\hline Diversión & $4,65 \pm, 35$ & $4,67 \pm, 55$ & $4,52 \pm, 55$ & $4,59 \pm, 57$ \\
Satisfacción & $3,99 \pm, 99$ & $4,20 \pm, 68$ & $3,90 \pm, 94$ & $4,43 \pm, 70$ \\
Autovalía & $4,3 \pm, 44$ & $4,52 \pm, 49$ & $4,03 \pm, 78$ & $4,58 \pm, 35$ \\
Autonomía & $3,9 \pm, 58$ & $3,96 \pm, 75$ & $3,70 \pm, 84$ & $4,09 \pm, 55$ \\
Competencia & $4,35 \pm, 55$ & $4,43 \pm, 76$ & $4,13 \pm, 75$ & $4,69 \pm, 36$ \\
Relaciones & $4,21 \pm, 63$ & $4,68 \pm, 45$ & $4,35 \pm, 73$ & $4,61 \pm, 53$ \\
Tarea & $4,64 \pm, 39$ & $4,67 \pm, 62$ & $4,58 \pm, 47$ & $4,84 \pm, 24$ \\
Ego & $2,74 \pm 1,04$ & $3,16 \pm 1,11$ & $3,02 \pm 1,17$ & $2,78 \pm 1,14$ \\
Atención & $4,11 \pm 1,65$ & $4,98 \pm 1,84$ & $4,56 \pm 1,37$ & $5,27 \pm 1,91$ \\
\hline Notas. Media \pm desviación típica & &
\end{tabular}

En cuanto a las diferencias intragrupos, no se presentan diferencias significativas entre la mayoría de las variables. En el grupo de intervención aparecen diferencias en cuanto a la variable de Autonomía, siendo ésta la única que se encuentra en el grupo de intervención. Asimismo, el grupo de control presenta un aumento significativo en la variable de Atención y no en las demás variables.

Tabla 3.- Estadísticos descriptivos pre-test y post-test

Variables

\begin{tabular}{lcrrrrrrrrr} 
& Div. & \multicolumn{1}{c}{ Satis. } & Autov & Auton & Comp & Rel. & Tar & Ego & Aten \\
\hline GC pre-post &, 594 &, 252 &, 369 &, 844 &, 281 &, 862 &, 412 &, 987 &, $019^{*}$ \\
GE pre-post &, 770 &, 316 &, 839 &, $016^{*}$ &, 136 &, 807 &, 725 &, 584 &, 237 \\
\hline Pre-test GC-GE &, 240 &, 223 &, 053 &, 187 &, 149 &, 386 &, 550 &, 284 &, 217 \\
Post-test GC-GE &, 914 &, 201 &, 337 &, 434 &, 515 &, 943 &, 080 &, 806 &, 877 \\
\hline
\end{tabular}

Notas. GC: grupo control; GE: grupo experimental; * $p<.05$ 
Independientemente a estos resultados, en cuanto a la comparación intergrupal, se observa que en ningún momento se establecen estadísticamente significativas entre las variables estudiadas.

\section{DISCUSIÓN}

Los objetivos que se plantearon fueron diseñar un programa de intervención de mindfulness en tercer ciclo de primaria, analizar la relación entre variables motivaciones y psicológicas, especialmente en educación física y comprobar los cambios después de la intervención.

Los audios se escogieron en función del contexto en los que se iban a llevar a cabo, es decir, adaptados a la etapa de educación primaria. Autores como Snel (2013), Lantieri y Goleman (2009) indican de la importancia de la utilización de los audios para realizar mindfulness, un elemento imprescindible para ello. La selección de los audios salió de los libros "la Inteligencia Emocional infantil y juvenil. Ejercicios para cultivar la fortaleza interior en niños y jóvenes" de Lantieri, y Goleman (2009) y "Tranquilos y atentos como una rana" de Snel (2013).

La relación entre las variables analizadas, se observa que la orientación a la tarea correlaciona positivamente con el resto. El clima motivacional que implica a la tarea se encuentra relacionado positivamente con las metas orientadas a la tarea, la diversión, la satisfacción, el interés y la motivación intrínseca (Almagro, 2012; Moreno Murcia et al,.(2008). Según Nicholls (1984), las dos perspectivas de orientación de metas (Ego y Tarea) se han mostrado ortogonales desde una perspectiva estadística por lo que cada individuo presenta las dos orientaciones, pudiendo variar en la intensidad del patrón motivacional (ego y tarea simultáneamente). Es decir que una persona puede, de forma independiente, tener una alta o baja orientación al ego y una alta o baja orientación a la tarea. Como podemos comprar con los resultados en el apartado de ego y tarea.

En este estudio se pretendía con la práctica de mindfulness dirigir la atención al momento presente que estábamos viviendo, por lo que lleva a evitar respuestas impulsivas que derivan mayormente de patrones negativos, siguiendo a autores como Ramos, Hernández y Blanca (2009). Así, actuando como Simón (2007) sería ésta actitud propia de la Atención Plena, la única maniobra que libera a la conciencia de la servidumbre de la emoción. Así, estaríamos ante el poder del control de nuestras emociones. Recientes investigaciones dentro del contexto escolar, ya mencionadas, muestran que a medida que el alumnado va aprendiendo a manejar sus competencias emocionales, se observa en su comportamiento las consecuencias positivas que esto pueda tener y además, evidencian que las competencias emocionales pueden contribuir de forma positiva al bienestar personal y social del alumnado (Bisquerra y Pérez, 2007). Otros autores tales como, Extremera y Fernández-Berrocal (2003), afirman que los alumnos emocionalmente inteligentes, como norma general, poseen mejores niveles de ajuste psicológico y bienestar emocional; presentan una mayor calidad y cantidad de redes interpersonales y de apoyo social; son menos propensos a 
realizar comportamientos disruptivos, agresivos o violentos; pueden llegar a obtener un mayor rendimiento escolar al enfrentarse a las situaciones de estrés con mayor facilidad. Por lo tanto, el saber controlar nuestras emociones amplía nuestro grado de bienestar personal y es a través de la práctica directa de 'Atención Plena' un camino para conseguirlo. La falta de resultados en el bienestar en nuestro estudio tras la intervención puede deberse al escaso tiempo empleado, así como a la edad del alumnado. Autores como Ramos, Recondo y Enríquez (2008) crearon el 'Programa de Entrenamiento de la Inteligencia Emocional Plena (PINEP), con el fin de aprender a gestionar los estados emocionales a través de un entrenamiento específico para aprender a gestionar emociones con la práctica de mindfulness. Los resultados obtenidos demostraron la valía de mindfulness como instrumento para mejorar la satisfacción vital y la realidad emocional en niños preadolescentes. A su vez, han señalado al programa como una herramienta útil en el refuerzo, guía y desarrollo de una empatía sana en niños, especialmente en su dimensión emocional, así como para la mejora de la atención con la disminución de pensamientos intrusivos y recurrentes. En otro estudio realizado por Franco, Fuente, y Salvador, (2011) se analizan los efectos que un programa de entrenamiento en mindfulness produjo sobre el sentimiento de crecimiento y autorrealización personal en adolescentes estudiantes de Bachillerato. Se realizó un diseño controlado y aleatorizado con un grupo experimental y un grupo control en lista de espera con medición pretest-postest, utilizando como instrumento de medida el Cuestionario de Autoconcepto y Autorrealización (AURE). Los resultados obtenidos muestran la existencia de diferencias significativas entre las puntuaciones postest-pretest. Se concluye que un programa de meditación, centrado en el entrenamiento de la conciencia plena (mindfulness), puede ser un instrumento válido y adecuado para promover el crecimiento y autorrealización personal. Seguimos encontrando evidencia sobre estudios que muestran la eficacia de las técnicas de meditación en la intervención y mejora de los niveles de autoconcepto, autoestima (De la Fuente, Salvador, y Franco, 2010), las habilidades sociales (De la Fuente, Franco, y Salvador, 2010) y en diversas variables personales: impulsividad, evitación experiencial, ansiedad social y estados de ánimo actuales (Franco, Fuente, y Salvador, 2011). Se podría decir que nuestros objetivos a pesar de los resultados obtenidos se encuentran en concordancia con lo planteado por Franco, Fuente y Salvador (2011) quienes afirman que se puede conseguir un mayor nivel de autorrealización mediante la práctica de la meditación y corroboran que las personas autorrealizadas se interesan por el bienestar de los demás, al tiempo que disfrutan de la interpersonalidad y de las relaciones de grupo, suelen disfrutar de las acciones como fines o placeres en sí mismas, son conscientes de sus propios sentimientos sin tratar de reprimirlos, muestran una autoaceptación incondicional, toleran las dificultades y las ambigüedades, son flexibles y abiertos al cambio, y muestran interés social y conductas altruistas.

Con relación a la diversión, tras analizar los resultados de este artículo podemos corroborar que los alumnos conciben la práctica del mindfulness como una actividad divertida y satisfactoria. Esto podemos comprobarlo en la tabla 1 de los resultados, donde se puede observar como alcanza una media muy alta, alcanzando valores similares al clima orientado a la tarea. 
Los resultados obtenidos muestran una mejoría en el perfil atencional de los participantes después de haber practicado la técnica mindfulness diariamente. En el grosor cortical se encuentran las regiones del cerebro implicadas en la atención, entre otras. Un estudio de Lazar, Kerr, Wasserman y Gray (2005) en el que sus participantes con formación y experiencia en Insight (un método de meditación, al igual que el mindfulness) practicaban entre 6,2 $\pm 4,0$ horas por semana. Los resultados obtenidos fueron significativos ya que aumentó el grosor cortical del hemisferio derecho, que es el encargado de mantener la atención (Lazar, Kerr, Wasserman, y Gray 2005). Por lo tanto, el mindfulness al ser otra técnica de meditación-relajación puede influir en el grosor cortical. Existen programas de formación para el entrenamiento de la atención, Jha, Krompinger, y Baime (2007) encontraron mejoras en la atención a través de un programa de mindfulness para la reducción del estrés, así mismo se encontraron mejoras en personas que lo practicaron de forma intensiva a corto plazo. Por lo tanto, el mindfulness a corto plazo tiene influencia en la atención plena en cualquier ámbito si se practica de forma intensiva, aunque si su práctica permanece durante el tiempo de forma menos intensiva las mejoras serán mayores y más duraderas. Así mismo, Toga (2012) en su estudio coincide con el estudio anterior en el cual a través de la meditación modifica algunos segmentos cerebrales mejorando la habilidad de procesamiento en las personas, por lo tanto, mejora su atención. La literatura científica muestra algunos estudios sobre el mindfulness como mejora de la atención en personas con déficit de atención con hiperactividad TDAH (Carboni, 2012). Los resultados muestran mejoras significativas en la atención en sus participantes. Un programa de Aristizábal y Dieste en el que se aplicó una combinación de yogamindfulness de una sesión semanal durante 8 semanas en participantes con TDAH mostró igualmente mejoras en el desarrollo de la "atención plena" así como mejora en otras funciones de procesamiento y mejoras en la implicación.

La atención se relaciona a la vez con la orientación a la tarea como se puede observar en los resultados obtenidos, esta mejora se debe a la habilidad de procesamiento que se ha desarrollado a través de la intervención. Esta relación puede conllevar a un mejor rendimiento en el ámbito escolar como concluye un estudio de León (2009) en el que la mejora de la atención a través del entrenamiento de la atención obtiene resultados significativos en el rendimiento utilizando una "Escala de atención plena en el ámbito escolar". En un estudio de Martín López, León del Barco y Vicente Castro (2007) similar al nuestro con participantes de primaria, a través de una intervención con técnica de relajación y atención interior encontró mejoría en algunos ámbitos escolares como aptitudes musicales y atención auditiva. Resultados similares se puede encontrar en otro estudio en el que relaciona la mejora del desempeño escolar a una intervención de mindfulness (Chang y Hierbert, 1989). Por lo contrario en nuestro estudio no se encontraron mejorías significativas en el rendimiento académico. Esto se puede deber al corto plazo en el que se aplicó la intervención o la calidad con la que se ha hecho la intervención diariamente. 


\section{CONCLUSIONES}

Después de analizar los resultados y fundamentar los mismos con otros estudios anteriores podemos afirmar que el programa de intervención de mindfulness ha ayudado a la mejora del perfil atencional del alumnado a los que se le ha aplicado. También promueve variables de crecimiento personal, mejora de nuestro concepto, es decir contribuye a nuestro bienestar y autovalía.

Analizadas las relaciones entre las diferentes variables estudiadas, comentar que en su mayoría se encuentran relacionadas positivamente. La variable que se puede relacionar con casi todas las demás es el clima motivacional que implica a la tarea ya que se atañe positivamente con las metas orientadas a la tarea, la diversión, la satisfacción, el interés y la motivación intrínseca. La atención se relaciona a la vez con la orientación a la tarea como se puede observar en los resultados obtenidos, esta mejora se debe a la habilidad de procesamiento que se ha desarrollado a través de la intervención

Como beneficios más relacionados con nuestro tema destacamos la mejora: del autoconocimiento y la regulación de las emociones; de la concentración y la resolución de dificultades al enfocar la experiencia en el "proceso" y no tanto en el "resultado" de nuestras acciones; y de las relaciones interpersonales, la empatía, la compasión y el clima del aula.

Recomendamos que la intervención de mindfulness dure más tiempo (varios meses o todo el curso) y quizás la edad del alumnado ha sido otro factor por el que los resultados en el pretes-postest hayan sido escasos.

En cualquier caso es necesario conocer la importancia de controlar nuestras emociones para ampliar nuestro grado de bienestar personal y a través de la práctica directa de 'Atención Plena' es posible conseguirlo.

\section{REFERENCIAS}

Almagro, B. J. (2012) Factores motivacionales relacionados con la adherencia a la práctica deportiva competitiva en adolescentes. Tesis Doctoral. Universidad de Huelva.

Atienza, F. L., Pons, D., Balaguer, I., y García-Merita, M. L. (2000). Propiedades psicométricas de la Escala de Satisfacción con la Vida en adolescentes. Psicothema, 12 (2), 331-336.

Balaguer, I.; Castillo, I., y Duda, J. (2008). Apoyo a la autonomía, satisfacción de las necesidades, motivación y bienestar en deportistas de competición: un análisis de la teoría de autodeterminación. Revista de Psicología del Deporte, 17, 123-139.

Bisquerra, R., y Pérez, N. (2007). Las competencias emocionales. Educación XXI, 10, 61 82. 
Carboni, J. (2012). The Impact of Mindfulness Training on Hyperactive Behaviors Demonstrated by Elementary Age Children with a Diagnosis of Attention Deficit Hyperactivity Disorder.

Davidson, R. J. (2012). El perfil emocional de tu cerebro. Claves para modificar nuestras actitudes y reacciones. Barcelona: Destino.

Chang, J. y Hierbert, B. (1989). Relaxation procedures with children: A review. Medical Psycotherapy: An International Journal, 22, 163-173.

De la Fuente, M.; Franco, C., y Salvador, M. (2010). Efectos de un programa de meditación (mindfulness) en la medida de la alexitimia y las habilidades sociales. Psicothema 2010. Vol. 22, no 3, pp. 369-375

Extremera, N. y Fernández-Berrocal, P. (2003). La inteligencia emocional en el contexto educativo. Revista de educación, 332, 97-116.

Franco, C., Fuente, M., y Salvador, M. (2011). Impacto de un programa de entrenamiento en conciencia plena (mindfulness) en las medidas de crecimiento $y$ autorrealización personal. Psicotema, 58-65

Fulton, R. P. y Sieguel, D. R. (2005). Buddhist and western psychology: Seeking commond ground. En C.K. Germer, R. D. Siegel y P. R. Fulton (Eds.), Mindfulness and Psychotherapy (pp. 28-51). Nueva York: Guilford Press.

García Calvo, T. (2004). La motivación y su importancia en el entrenamiento con jóvenes deportistas. Memoria de Docencia e Investigación. Cáceres: Facultad de Ciencias del Deporte, Universidad de Extremadura.

Jha, A., Krompinger, J., y Baime, M. (2007). Mindfulness training modifies subsystems of attention. Cognitive, Affective, \& Behavioral Neuroscience, 7(2), 109-119.

Kabat- Zinn (1994). Wherever you go, there you are: Mindfulness meditation in everyday life. New York: Hyperion.

Káiser, S. (2013). El niño atento mindfulness para ayudar a tu hijo a ser más feliz, amable y compasivo. Desclée de brouwer S.A., Bilbao.

Lantieri, L., y Goleman, D. (2009). Inteligencia Emocional infantil y juvenil. Ejercicios para cultivar la fortaleza interior en niños y jóvenes.Santillana. Madrid.

Lazar, S., Kerr, C., Wasserman, R., y Gray, J. (2005). Meditation experience is associated with increased cortical thickness. Neuroreport.

León, B. (2009). Atención plena y rendimiento académico en estudiantes de enseñanza secundaria. European Journal of Education and Psychology, 1(3), 17-26

Lutz, A., Rawlings, N., y Davidson, R. J. (2005). Changes in the tonic high-amplitude gamma oscillations during meditation correlates with long-term practitioners' verbal reports. Paper presented at the Society for Neuroscience, Washington, DC.

Mañas, I. M. (2009). Mindfulness (Atención Plena): La meditación en Psicología Clínica. Gaceta de Piscología, 13-29

Martín López, E., León del Barco, B., \& Vicente Castro, F. (2007). Mejora de las aptitudes musicales mediante una intervención en atención auditiva e interior. Revista galego-portuguesa de Psicoloxía e Educación, 14, 95-105.

Moñiva, A., García-Diex G., y García-de-Silva, R. (2012). Mindfulness (atención plena). Concepto y teoría. Portularia Vol 12 № extra (pp 83-89).

Moreno, J. A.; González-Cutre, D.; Chillón, P. y Parra, D. (2008). Coeducación y climas de aprendizaje en educación física. Aportaciones desde la teoría de Metas de Logro.International Journal of Sport Science, 11, (pp. 42-64). Consultada el 4/7/2014 en http://dialnet.unirioja.es/servlet/articulo?codigo=2549908 
Moreno, J. A., López, M., Martínez Galindo, C. M., Alonso, N., y González-Cutre, D. (2007). Validación preliminar de la escala de percepción del clima motivacional de los iguales (CMI) y la escala de las orientaciones de meta en el ejercicio (GOES) con practicantes españoles de actividades físico-deportivas. Revista Iberoamericana de Psicología del Ejercicio y el Deporte, 1(2), 13-28.

Nyanaponika T. (1962). The heart of Buddhist meditation: A handbook of mental training based on the Buddha's way of mindfulness. London: Rider and Company

Nicholls, J. (1984). Conceptions of ability and achievement motivation. In R. Ames and C. Ames (Eds.), Research on motivation in education: Student motivation (pp. 39-73). New York, Academic Press.

Ramos, N.S., Hernández, S.M., y Blanca, M.J. (2009). Efecto de un programa integrado de mindfulness e inteligencia emocional sobre las estrategias cognitivas de regulación emocional. Ansiedad y Estrés, 15, 207-216

Ramos, N., Recondo, O, y Henríquez, H. (2012). Practica la Inteligencia Emocional Plena: la gestión eficaz de las emociones a través de mindfulness. Barcelona: Ed Kairós.

Sánchez Gutiérrez, G. (2011). Meditación, mindfulness y sus efectos biopsicosociales. Revisión de literatura. Revista Electrónica de Psicología de Iztacala, 223-254

Siegel, D. J. (2010). The mindful therapist. New York: Norton and Company.

Silananda, U. (1990). The four foundations of mindfulness. Boston: Wisdom Publications.

Simón (2007). Mindfulness y Neurobiology. Revista de Psicoterapia, XVII (66-67); 5-30

Snel, E. (2013). Tranquilos y atentos como una rana. Kairos, S.A. Barcelona.

Sogyal, R. (1994). El libro tibetano de la vida y de la muerte. Barcelona: Urano.

Toga, E. (2012). The unique brain anatomy of meditation practitioners: alterations in cortical gyrification, Frontiers in Human Neuroscience, 1-9.

Vallejo, M. A. (2006). Mindfulness. Papeles del Psicólogo, 27, 92-99. 